\title{
Einfluss der Geometrie von Elektroden und Sensorschichten auf das Schwingungsverhalten von Langasit-Resonatoren
}

\author{
Silja Schmidtchen, Han Xia und Holger Fritze
}

Institut für Energieforschung und Physikalische Technologien, Technische Universität Clausthal, Am Stollen 19B, 38640 Goslar

\section{Zusammenfassung}

Auf dem hochtemperaturstabilen piezoelektrischen Material Langasit basierende Resonatoren können als Gassensoren genutzt werden, indem die Auswirkung von Änderungen der elektrischen und mechanischen Eigenschaften einer aufgebrachten Sensorschicht auf das Schwingungsverhalten detektiert werden. Als Messgröße wird hierbei nicht nur die massebedingte Resonanzfrequenzverschiebung sondern auch die Veränderung des Schwingungsprofils durch Änderung des effektiven Elektrodendurchmessers genutzt. Um die letztgenannte Größe zu variieren, werden Metalloxidschichten auf den Resonator aufgebracht, deren Leitfähigkeit sich mit sinkendem Sauerstoffpartialdruck erhöht. Die Untersuchung der leitfähigkeitsinduzierten Änderung des effektiven Elektrodendurchmessers und des Einflusses auf die räumliche Verteilung der mechanischen Auslenkung des Resonators erfolgt mit einem Laser DOPPLER Vibrometer bei hohen Temperaturen und verschiedenen Sauerstoffpartialdrücken. Damit werden Informationen über die Ortsabhängigkeit der Massenempfindlichkeit zugänglich, da diese Größe direkt proportional zur Schwingungsamplitude ist [1].

\section{Material}

Langasit $\left(\mathrm{La}_{3} \mathrm{Ga}_{5} \mathrm{SiO}_{14}\right.$, LGS) ist ein einkristallines piezoelektrisches Material mit derselben Raumgruppe wie $\alpha$-Quarz. Im Vergleich zu Quarz und anderen herkömmlichen Piezoelektrika ist Langasit thermisch stabil und lässt sich bis zu Temperaturen nahe des Schmelzpunktes bei $1470{ }^{\circ} \mathrm{C}$ zu Volumenschwingungen anregen [2]. Der atomare Transport in Langasit, wie auch die Stabilität bei hohen Temperaturen wurden in vorangegangenen Arbeiten ausführlich untersucht und Einsatzgrenzen dieses Materials bestimmt [3]-[6].

\section{Sensorprinzip}

Der Sensor basiert auf Langasit-Resonatoren. Das Resonanzverhalten ist abhängig von verschiedenen externen Einflüssen, wie z. B. Massenbeladung, und somit als Sensormessgröße nutzbar. Dies wird seit langem bei Mikrowaagen genutzt, um z. B. Beschichtungsprozesse zu überwachen. Alternativ können Sensorschichten auf den Resonator aufgebracht werden, um Aussagen über atmoshärenbedingte Änderungen von Schichteigenschaften, wie Masse, Steifigkeit oder Leitfähigkeit und somit über die umgebende Atmosphäre zu treffen. Abhängig von der Geometrie der Elektroden und der Schicht kann man dabei verschiedene Sensoreffekte nutzen [7]-[8]. In Bild 1a ist das Prinzip eines im Mikrowaagemodus betriebenen Gassensors dargestellt. Auf die Vorder- und Rückseite eines Resonators werden unterschiedlich große, schlüssellochförmige Platin-Elektroden aufgebracht. Die Sensorschicht befindet sich auf der größeren Elektrode. Somit kann eine Änderung der Schichtmasse in Form einer Verschiebung der Resonanzfrequenz detektiert werden. Da die Sensorschicht den Bereich der Elektrode nicht überschreitet, ist die induzierte Frequenzänderung unabhängig von einer Änderung der Schichtleitfähigkeit. Das Resonanzverhalten ist also nur von den mechanischen Eigenschaften der Schicht abhängig.

Im Leitfähigkeitsmodus (Bild 1b) werden ebenfalls verschieden große Elektroden auf den Resonator aufgebracht. Die Sensorschicht wird hier jedoch auf der kleineren Elektrode abgeschieden und überlappt 
diese. Durch eine Änderung der Schichtleitfähigkeit verändert sich somit der effektive Durchmesser der Elektrode. Das Schwingungsprofil eines Scherwellen-Resonators und damit seine Massenempfindlichkeit werden hauptsächlich durch die Geometrie der Elektroden und der aufgebrachten Schicht bestimmt. Im Bereich der Elektroden wird der Resonator mit einer Resonanzfrequenz zu Schwingungen angeregt, die durch die Dicke der Elektroden inklusive der Sensorschicht bestimmt wird. Außerhalb der Elektroden ist die Dicke geringer, was zu einer höheren Resonanzfrequenz führt [1]. Folglich kann sich die Schwingung nicht beliebig über den Rand der Elektroden ausbreiten. Die Schwingungsenergie ist stark auf diesen Bereich eingegrenzt (Energy-Trapping). Die räumliche Verteilung der Schwingungsamplitude $x(r)$ und die Massenempfindlichkeit $S(r)$ können durch eine GAUSS-Verteilung $S(r)=S_{\max } e^{-w r^{2}}$ angenähert werden. Das Maximum befindet sich dabei in der Mitte der Elektrode [9]. Die Breite des GAUss-Profils ist vom Radius der Elektroden $r_{e}$ entsprechend

$$
w=\frac{\ln \left(S_{e l} / S_{\max }\right)}{r_{e}^{2}}
$$

abhängig. Eine Vergrößerung der Elektrodenfläche durch eine zunehmende Leitfähigkeit der Sensorschicht führt somit zu einer deutlichen Verbreiterung der Verteilung der Massenempfindlichkeit (vgl. Bild 2).

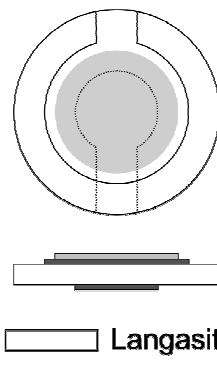

(a)
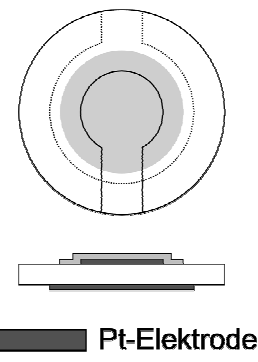

Sensorschicht

(b)

Bild 1 Layouts der verschiedenen Sensorgeometrien. (a) Mikrowaagemodus (b) Leitfähigkeitsmodus

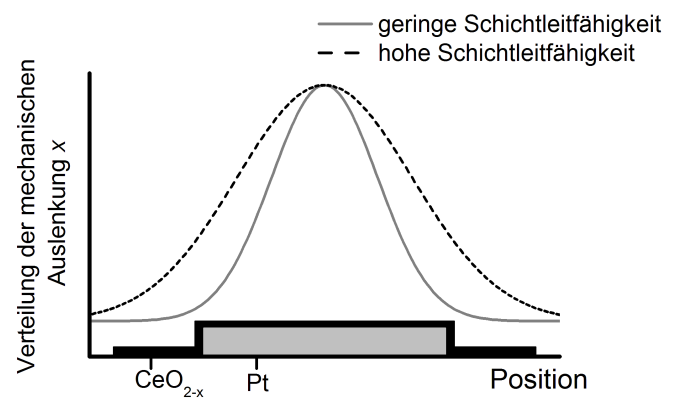

Bild 2 Veränderung des Schwingungsprofils eines Resonators mit der Schichtleitfähigkeit

\section{Experimenteller Aufbau und Messungen}

Der hier benutzte Dickenscherschwinger ist ein scheibenförmiger Langasit-Resonator (y-Schnitt) mit einem Durchmesser von $19 \mathrm{~mm}$ und einer Dicke von $600 \mu \mathrm{m}$. Die Resonanzfrequenz des Grundtons beträgt 2,2 MHz. Auf der Vorder- und Rückseite des Resonators sind Platin-Elektroden wie oben erläutert aufgebracht. Die kleinere der beiden Elektroden wird mit Hilfe der Laserablation abgeschieden und hat einen Durchmesser von 5,8 mm bzw. eine Dicke von $300 \mathrm{~nm}$. Für die Messungen mit dem Laser DOPPLER Vibrometer ist eine hohe Streuung des Laserlichts an der Oberfläche des zu messenden Objektes erforderlich, um eine ausreichende Intensität des zurückgestreuten Strahls zu gewährleisten. Daher wird die größere Elektrode (Durchmesser 10,3 mm) im Siebdruckverfahren hergestellt, was eine hohe Rauhigkeit und somit gute Streuung des Lichts gewährleistet (vgl. Bild 3). Die Dicke der Siebdruck Elektrode beträgt ungefähr $3,0 \mu \mathrm{m}$. Da es sich bei den Proben um Dickenscherschwinger mit einer Auslenkung in Richtung der Probenoberfläche (x-Richtung des Kristalls) handelt, muss die Probe geneigt eingebaut werden (Bild 3). Bei der Auswertung werden die Auslenkungen entsprechend des hier gewählten Neigungswinkels von $35^{\circ}$ korrigiert.

Um eine Veränderung des Elektrodendurchmessers zu realisieren, wird eine zusätzliche Oxidschicht, in diesem Fall Ceroxid $\left(\mathrm{CeO}_{2-\mathrm{x}}\right)$, über der kleineren Elektrode mit Laserablation aufgebracht. Der Durchmesser ist mit 13,2 mm größer als der der Metallelektrode (vgl. Bild 1b). Eine Verringerung des Sauerstoffpartialdrucks führt zu einer Änderung der Stöchiometrie der Ceroxid-Schicht und somit zur Erhöhung der Leitfähigkeit.

Mit dem Vibrometer wird bei Raumtemperatur und -luft die räumliche Verteilung der Schwingungsamplitude für den Grundton und einen Oberton mit linearen Scans gemessen. Weiterhin werden Raster- 
Scans an der Probe durchgeführt. Hierbei wird das Schwingungsprofil auf der Elektrodenfläche mit 100 x 100 Messpunkten aufgenommen.

Weitere Messungen werden in einem gasdichten Ofen bei $580{ }^{\circ} \mathrm{C}$ bei durchgeführt. Zur Variation des Sauerstoffpartialdrucks wird ein Gasmischsystem genutzt. Der eingestellte Wert wird mittels eines Zirkonoxid-basierten Sauerstoffsensors gemessen. Das Laser DOPPLER Vibrometer (Polytec OFV 5059) ist außerhalb des Ofens vor einem gasundurchlässigen Sichtfenster in einem Arbeitsabstand von ca. 0,5 m positioniert.

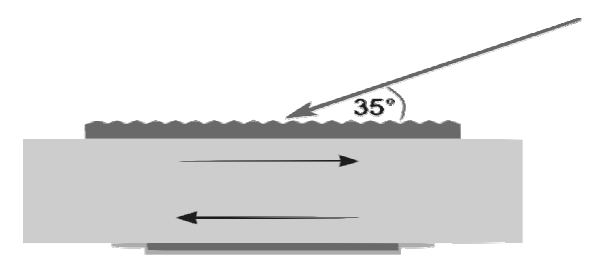

Bild 3 Schematische Darstellung eines Resonators mit rauer Siebdruckelektrode und einfallendem Laserstrahl

Auf der Oberfläche der Probe wird ein linearer Scan mit einer Schrittweite von ca. $250 \mu \mathrm{m}$ durchgeführt. Für eine Temperatur von $580{ }^{\circ} \mathrm{C}$ und drei verschiedene Sauerstoffpartialdrücke, $10^{-4}, 10^{-16}$ und $10^{-20}$ bar, wird die mechanische Auslenkung des Resonators an 50 verschiedenen Positionen gemessen. Die Amplitudenverteilungen werden mit einer GAUSS-Funktion gefittet.

\section{Ergebnisse}

Bild 4a zeigt die aus den linearen Scans ermittelte mechanische Auslenkung. Für den Grundton ergibt sich ein signifikant breiteres Schwingungsprofil als für den Oberton. Die Ergebnisse des Raster-Scans über die Fläche der Elektroden ist in Bild 4b gezeigt. Der GAUSS-förmige Verlauf des Schwingungsprofils ist deutlich zu erkennen.

(a)

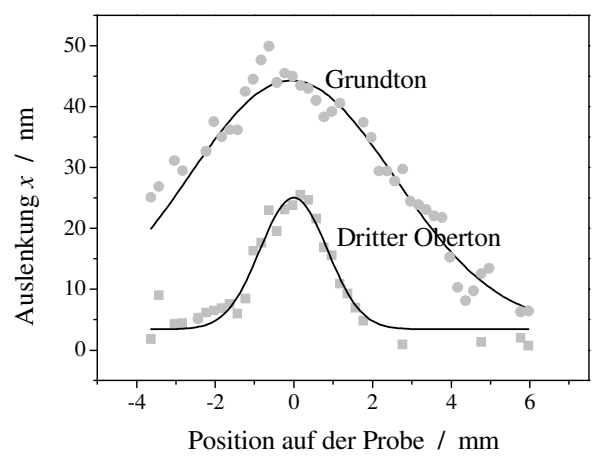

(b)

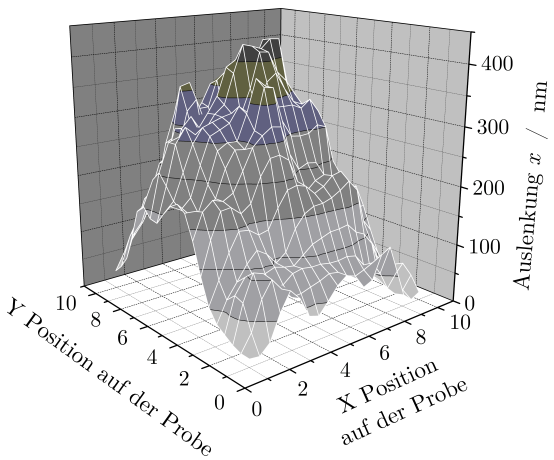

Bild 4 (a) Räumliche Verteilung der Auslenkung eines Resonators, angeregt mit den Resonanzfrequenzen des Grundtons (ca. $2 \mathrm{MHz}$ ) und eines Obertons (ca. $6 \mathrm{MHz}$ ) bei Raumtemperatur. Anregungsspannung $\mathrm{U}_{\mathrm{A}}=2 \mathrm{~V}$ für den Grundton und $5 \mathrm{~V}$ für den Oberton. (b) Raster-Scan eines Ceroxid-beschichteten Langasitresonators in 3D-Auftragung, $\mathrm{U}_{\mathrm{A}}=9 \mathrm{~V}$.

Bei Messungen in reduzierenden Atmosphären und einer Temperatur von $580{ }^{\circ} \mathrm{C}$ (Bild 5b) lässt sich eine Verbreiterung des Schwingungsprofils mit abnehmendem Sauerstoffpartialdruck bestimmen. Da Langasit bei $580{ }^{\circ} \mathrm{C}$ praktisch keine Änderung der Leitfähigkeit als Funktion des Sauerstoffpartialdrucks bis zu einem Wert von $10^{-21}$ bar aufweist, sind die Änderungen des Resonanzverhaltens im Leitfähigkeitsmodus (Bild 1a) auf eine Verringerung des Schichtwiderstandes zurückzuführen. Es ergibt sich eine Vergrößerung der effektiven Elektrodenfläche um $18 \%$, was einer Zunahme des Durchmessers um den Faktor 1,09 entspricht. Die Verbreiterung der Schwingungsverteilung folgt indirekt auch aus in früheren Arbeiten durchgeführten elektrischen Messungen (siehe hierzu [7]). Das elektrische Verhalten eines Resonators wird hier mit einem Butterworth-van-Dyke-Ersatzschaltbild angenähert. Die Vergrößerung der Elektrodenfläche spiegelt sich in den Parametern des Ersatzschaltbildes wider. Insbesondere nehmen die Volumenkapazität zu sowie der Volumenwiderstand und die Bewegungskapazität ab. Letzteres entspricht einer Zunahme der effektiven Elektrodenfläche um $15 \%$ (Durchmesservergrößerung um Faktor 1,07). 


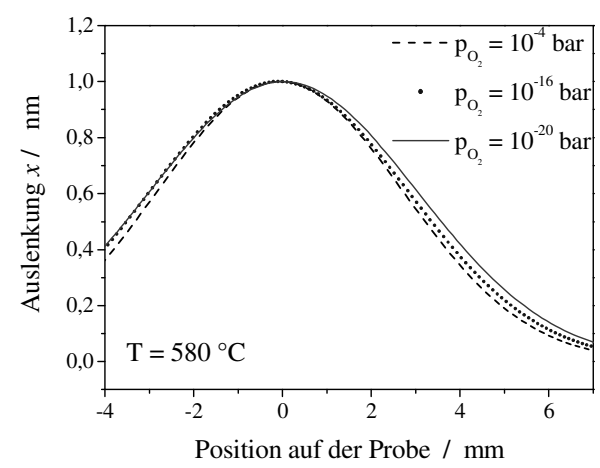

Bild 5 Schwingungsprofil eines Resonators mit $\mathrm{CeO}_{2-\mathrm{x}}$-Schicht bei $580{ }^{\circ} \mathrm{C}$ und drei verschiedenen Sauerstoffpartialdrücken.

\section{Fazit}

Messungen der räumlichen Verteilung der Schwingungsamplitude eines Langasit-Resonators werden mit einem Laser DOPPLER Vibrometer bei Temperaturen bis $580{ }^{\circ} \mathrm{C}$ durchgeführt. Beim Vergleich der Schwingungsprofile des Grundtons und eines Obertons ergeben sich eine deutlich geringere Amplitude und ein schmaleres Schwingungsprofil für den Oberton. Dies ist bei der Berechnung der Massensensitivität eines bei einem Oberton betriebenen Resonators zu berücksichtigen. Weiterhin wurde der Einfluss der effektiven Elektrodengröße auf das Schwingungsprofil gezeigt. Eine Verbreiterung des Profils ergibt sich mit Verringerung der Schichtleitfähigkeit durch einen abnehmenden Sauerstoffpartialdruck in der Umgebung. Die Informationen über die Veränderung des Schwingungsprofils eines Resonators erlaubt es, die Geometrie von Sensorschicht und Metallelektrode zu optimieren und somit verbesserte Gassensoren für den hohen Temperaturbereich zu schaffen.

\section{Literatur}

[1] G. Sauerbrey, Verwendung von Schwingquarzen zur Wägung dünner Schichten und zur Mikrowägung, Zeitschrift für Physik 155, 1959, S. 206-222.

[2] D. Damjanovic, Materials for high temperature piezoelectric transducers, Curr. Opin. Solid State Mater. Sci. 3, 1998, S. 469.

[3] J. Hornsteiner, E. Born and E. Riha, Langasite for High Temperature Surface Acoustic Wave Applications, Phys. Stat. Sol. A 163, 1997, S. R3-R4.

[4] M. Schulz, D. Richter, J. Sauerwald and H. Fritze, Solid State Sensors for Selective Gas Detection at High Temperatures - Principles and Challenges, Integrated Ferroelectrics 115, 2010, S. 41-56.

[5] E. Ansorge, B. Schmidt, J. Sauerwald and H. Fritze, Langasite for microelectromechanical systems, Phys. Stat. Sol. A 208, 2011, S. 377-389.

[6] H. Fritze, High-temperature bulk acoustic wave sensors, Meas. Sci. Technol. 22, 12002, 2011, S. 28.

[7] H. Fritze, D. Richter and H. Tuller, Simultaneous detection of atmosphere induced mass and conductivity variations using high temperature resonant sensors, Sens. \& Act. B. Chemical, 111:112, 2005, S. 200-206.

[8] D. Richter, T. Schneider, S. Doerner, H. Fritze und P. Hauptmann, Selective Gas Sensor System for CO and $\mathrm{H}_{2}$ Distinction at High Temperatures Based on a Langasite Resonator Array, TRANSDUCERS 2007, S. 991-994.

[9] B. A. Martin und H. E. Hager, Velocity profile on quartz crystals oscillating in liquids, J. Appl. Phys. 65(7), 1989, S. 2630-2635. 